\title{
Improving Flow Property of Nifedipine Loaded Solid-Lipid Nanoparticles by Means of Silica for Oral Solid Dosage Form
}

\author{
Ranjan Kumar Barman1,2, Yasunori Iwao', Shuji Noguchi', Mir Imam Ibne Wahed2, \\ Shigeru Itai ${ }^{*}$ \\ ${ }^{1}$ Department of Pharmaceutical Engineering and Drug Delivery Science, School of Pharmaceutical Sciences, \\ University of Shizuoka, Shizuoka, Japan \\ ${ }^{2}$ Department of Pharmacy, Faculty of Science, University of Rajshahi, Rajshahi, Bangladesh \\ Email: ${ }^{*}$ s-itai@u-shizuoka-ken.ac.jp
}

Received 29 September 2014; revised 28 October 2014; accepted 18 November 2014

Academic Editor: Ravi K. Sajja, Texas Tech University Health Sciences Center, USA

Copyright (C) 2014 by authors and Scientific Research Publishing Inc.

This work is licensed under the Creative Commons Attribution International License (CC BY).

http://creativecommons.org/licenses/by/4.0/

(c) (i) Open Access

\begin{abstract}
In this study, a new formulation of silica nanocomposite containing nifedipine (NI) loaded freezedried solid-lipid nanoparticles (NI-SLNs) and silica have been developed with improved flowability of powders, which can lead to the formulation of a widely acceptable oral dosage form. The stable NI-SLNs were prepared using two phospholipids, hydrogenated soybean phosphatidylcholine and dipalmitoylphosphatidylglycerol mixed with $2.5 \% \mathrm{w} / \mathrm{v}$ trehalose as a cryoprotectant followed by lyophilization. We employed various grades of two types of silica, such as fumed and precipitated. Silica improved the poor flow property of NI-SLNs to good category as per USP-29. In addition, most of the silica nanocomposites showed the satisfactory results in their physicochemical properties such as particle size, polydispersity index, zeta potential, and recovered potency by around $100 \mathrm{~nm}, 0.3,-50 \mathrm{mV}$, and $80 \%$, respectively. Furthermore, it was found that NI-SLNs were easily released form nanocomposites within $30 \mathrm{~min}$, therefore, suggesting an improvement of drug dissolutions. Among them, precipitated silica cooperated fairly in improving the powder characteristics as well as the physicochemical, morphological, and pharmaceutical properties.
\end{abstract}

\section{Keywords}

Silica, Solid-Lipid Nanoparticle, Solid Dosage Form, Nifedipine, Flowability

\footnotetext{
${ }^{*}$ Corresponding author.
}

How to cite this paper: Barman, R.K., Iwao, Y., Noguchi, S., Wahed, M.I.I. and Itai, S. (2014) Improving Flow Property of Nifedipine Loaded Solid-Lipid Nanoparticles by Means of Silica for Oral Solid Dosage Form. Pharmacology \& Pharmacy, 5, 1119-1129. http://dx.doi.org/10.4236/pp.2014.512122 


\section{Introduction}

Oral drug delivery is the most convenient and preferred among all routes of drug administration. Of this oral delivery system, solid formulations, especially tablet, is the most acceptable, patient friendly and robust dosage form considering its favorable physico-pharmaceutical characteristics, low economic cost, simplicity in administration, and easiest transportation. When this dosage unit containing poorly water-soluble drugs is administered orally, insufficient dissolution and corresponding inadequate bioavailability might occur throughout the gastro-intestinal tract [1]. Developing strategies to improve drug dissolution rate and to enable the effective oral delivery for poorly water-soluble drugs is currently one of the greatest challenges to formulator in the pharmaceutical research field [2] [3]. Various approaches have been employed to tackle this limitation [4]-[9] and nano-based drug delivery systems have greater potential. This system have been applied for a variety of areas, such as multiple targeting, in vivo imaging, extended circulation time, and systemic control release, and it can be successfully used as drug delivery tools for currently available bioactive compounds [10]. Recently, lipid-based nanoparticulate systems such as microemulsions, solid-lipid nanoparticles (SLNs), and nanoemulsions have been proved as one of the useful approaches to improve solubility and absorption of drug [6].

SLNs, which are composed of the matrix of biocompatible solid lipids like triglycerides, fatty alcohols, phospholipids and waxes, have some advantages with respect to stability, drug release profile, and biocompatibility. Few limitations of SLN dispersions, however, include physical instability arising due to particle aggregation and gelation under accelerated storage conditions [11]. This drawback can be overcome by conversion from its dispersion to dry powder. Lyophilization has been the most common method to convert SLN dispersion to dry form [12]. Recently, we developed a newly formulated solid-lipid nanoparticle of nifedipine (NI-SLN) using only two solid phospholipids, e.g., hydrogenated soybean phosphatidylcholine (HSPC) and dipalmitoylphosphatidylglycerol (DPPG), without any emulsifier and organic solvent in the form of aqueous dispersion by high pressure homogenization technique. Although NI-SLN dispersions were stable for up to 4 months at $6^{\circ} \mathrm{C}$, a specialized storage condition [13], lyophilization technique with trehalose (a disaccharide) as a cryoprotectant enhanced stability for up to 6 months at $30^{\circ} \mathrm{C}$ and $65 \% \mathrm{RH}$ [14]. Moreover, no deterioration of the oral bioavailability of the encapsulated drug, along with hemocompatiblity was observed [15] [16]. However, considering conclusive oral formulation using this SLN, development of SLN powder with an adequate powder flow property is necessary because only powder of SLN with trehalose is easily expected to show low flowability.

Since 2001, silica-based nanoparticles have been recognized as a promising drug delivery system using porous silica, in particular, mesoporous material MCM-41 was firstly proposed [17]. Porous silica possesses several attractive features including high surface area and pore volume, good biocompatibility, chemical inertness as well as being able to modify the surface and pore size, affording control over the drug release [18]-[23]. The enhanced dissolution rate of drugs is based on the adsorption of drug molecules by Van der Waals forces and hydrogen bonds to the surface and pores of the silica carrier. The bonds can be easily broken when in contact with water and the drug molecules are consequently released. It is worth mentioning that despite the significant progress in the utilization of porous silica for drug delivery applications, there are only a few studies that demonstrate the silica formulations for lipid emulsions. For example, a versatile silica composite fabricated with lipid emulsion of celecoxib, a non-steroidal anti-inflammatory drug, have been reported to show its free flowing property satisfactorily after lyophilization, which might be employed to formulate an oral solid dosage form [24]. In another study, silica-lipid microparticles were reported to attain better control and predictability over drug release, solubilization, and in vivo pharmacokinetics [25] [26]. Furthermore, a surfactant free solid-lipid microparticle prepared by hot melt process and coated with various types of silica was also reported to modulate the kinetics of ibuprofen release [27]. Nevertheless, there have been no reports to date of a surfactant-free SLNs obtained by high pressure homogenization technique, of which the release behavior can be influenced in presence of porous silica, ultimately increasing the flow property of powdered nanoparticles. We assume that, as the physical state of lipid component in NI-SLNs is solid, unlike the lipid emulsion, the adsorption characteristics of SLNs on the surface of silica may differ from that of emulsion type NPs attributed to the different mechanisms in the formation of strong/weak chemical bonding. Therefore, a study of the functionality of porous silica on the drug release behavior along with its physicochemical characteristics of SLNs presents a major interest to promote the applications in formulating an oral solid dosage form.

In the present study, two types of hydrophilic silica named Aerosil ${ }^{\circledR}$ and Carplex ${ }^{\circledR}$ were employed to prepare silica nanocomposite (SNC) for NI-SLN because of their different characteristics such as particle size and pore 
size. NI-SLN was prepared by high pressure homogenization technique using two phospholipids, HSPC and DPPG, and lyophilized with different types of silica and trehalose, which was used as a cryprotectant [14]. The physicochemical, morphological, pharmaceutical, in vitro drug release characteristics were also investigated.

\section{Materials and Methods}

\subsection{Materials}

Hydrogenated soybean phosphatidylcholine [COATSOME ${ }^{\circledR}$ NC-21(HSPC)] and dipalmitoylphosphatidylglycerol [COATSOME ${ }^{\circledR}$ MGLS-6060 (DPPG)] were purchased from Nippon Oil and Fats Co., Ltd. (Tokyo, Japan). Nifedipine (JPXIV, NI) was provided by Nippon Fine Chemical Co., Ltd. (Osaka, Japan). Trehalose was purchased from Wako Pure Chemical Industries, Ltd. (Osaka, Japan). Silica (Aerosil ${ }^{\circledR}$ and Carplex ${ }^{\circledR}$ ) were kindly provided from Evonik Japan Industries Ltd. (Tokyo, Japan). The membrane filters (pore size: 0.20 and $0.45 \mu \mathrm{m}$ ) were purchased from Toyo Roshi Kaisha Ltd. (Tokyo, Japan). All reagents were of the highest grade commercially available and all solutions were prepared using de-ionized distilled water.

\subsection{Experimental}

\subsubsection{Preparation of Nifedipine (NI)-Loaded Solid-Lipid Nanoparticle Dispersion}

To prepare the NI-loaded solid-lipid nanoparticle dispersion (SLND), $40 \mathrm{mg}$ of NI and $1000 \mathrm{mg}$ of lipids (HSPC: DPPG, 5:1 molar ratio) were added to a mortar and physically mixed for 5 min. The mixture was then co-ground by a roll mill (R3-1R, Kodaira Seisakusho Co., Ltd., Tokyo, Japan). The co-grinding cycle was repeated 10 times. The resultant roll mixture was dispersed in $200 \mathrm{ml}$ of de-ionized distilled water and premixed using a Speed Stabilizer (10,000 rpm, Kinematica Co., Luzern, Switzerland) at $9000 \mathrm{rpm}$ for $10 \mathrm{~min}$, followed by high pressure homogenization (max pressure: $200 \mathrm{MPa}$, Microfluidizer ${ }^{\circledR}$, M110-E/H; Microfluidics, Co., Newton, MA, USA) with a pass cycle of 100 [28]. The suspension thus obtained was filtered through a $0.2 \mu \mathrm{m}$ membrane filter and stored in a refrigerator at $6^{\circ} \mathrm{C}$ until further use.

\subsubsection{Lyophilization}

To prepare lyophilized product, the SLND was collected into small vials followed by the addition of $2.5 \% \mathrm{w} / \mathrm{v}$ aqueous solution of trehalose (TS) at a ratio 1:1 (SLND: TS) and mixed well. Each vial was then frozen at $-40^{\circ} \mathrm{C}$ for $3 \mathrm{~h}$ and the frozen sample was freeze-dried in a glass chamber for $24 \mathrm{~h}$ using a vacuum pump accompanied by a vapor condenser $\left(-20^{\circ} \mathrm{C}, 0.0225\right.$ Torr). Then, secondary drying was carried out at $20^{\circ} \mathrm{C}$ for $24 \mathrm{~h}$. This lyophilized product was simply labeled as SLN and used as control.

\subsubsection{Preparation of Lyophilized Silica Nanocomposites (SNC) of Nifedipine Solid-Lipid Nanoparticle}

For the preparation of SNC, silica was well dispersed in water first by means of a magnetic stirrer in a beaker for 30 min. Again, both SLND and the silica dispersion (using silica-to-lipid ratio $=1: 9$ and 2:8) were agitated using a magnetic stirrer in a beaker for $30 \mathrm{~min}$ to allow the adsorption of nanoparticles by silica. This mixture (silica nanocomposite dispersion) equivalent to $2 \mathrm{ml}$ of SLND was transferred to small vials. TS at a concentration $2.5 \% \mathrm{w} / \mathrm{v}$ was added at a ratio 1:1 (SLND: TS) and mixed well. Each vial was then frozen at $-40^{\circ} \mathrm{C}$ for $3 \mathrm{~h}$ and the frozen sample was freeze-dried in a glass chamber for $24 \mathrm{~h}$ using a vacuum pump accompanied by a vapor condenser $\left(-20^{\circ} \mathrm{C}, 0.0225\right.$ Torr). Then, secondary drying was carried out at $20^{\circ} \mathrm{C}$ for $24 \mathrm{~h}$. These lyophilized SNC were labeled according to the first alphabet of the name of silica used and its concentration. For example, SLN-A200 (10\%) and SLN-C67 (20\%) are attributed to nanocomposite containing NI-SLNs with Aerosil-200 at silica-to-lipid ratio, 1:9, and with Carplex-67 at silica-to-lipid ratio, 2:8, respectively. The labels and compositions of the different SNCs used in this work are described in Table 1.

\subsection{Particle Characterization}

\subsubsection{Carr's Flow Ability Index and Hausner Ratio}

To measure the flow property, SNCs with a silica-to-lipid ratio of 2:8 were prepared as described in section 2.2.3. Flow ability of the powders were determined by following Carr's method [29]. Briefly, a measured quantity (about $0.5 \mathrm{~g}$ ) of powder passed through $1 \mathrm{~mm}$ sieve was taken in a 5 сc ( $0.1 \mathrm{cc}$ graduated) measuring cy- 
Table 1. Composition of silica nanocomposites.

\begin{tabular}{cccccc}
\hline Labeled name & Silica-to-lipid & Composition & & \\
& $\begin{array}{c}\text { NI solid-lipid nanoparticle } \\
\text { dispersion (ml) }\end{array}$ & Silica used & $\begin{array}{c}\text { Qty. of silica } \\
\text { (mg) }\end{array}$ & $\begin{array}{c}2.5 \% \text { w/v Trehalose } \\
\text { (ml) }\end{array}$ \\
\hline SLN & - & 2.0 & - & - & 2.0 \\
SLN-A200 (10\%) & $1: 9$ & 2.0 & Aerosil-200 & 1.11 & 2.0 \\
SLN-A200 (20\%) & $2: 8$ & 2.0 & Aerosil-200 & 2.50 & 2.0 \\
SLN-A380 (10\%) & $1: 9$ & 2.0 & Aerosil-380 & 1.11 & 2.0 \\
SLN-A380 (20\%) & $2: 8$ & 2.0 & Aerosil-380 & 2.50 & 2.0 \\
SLN-C67 (10\%) & $1: 9$ & 2.0 & Carplex-67 & 1.11 & 2.0 \\
SLN-C67 (20\%) & $2: 8$ & 2.0 & Carplex-67 & 2.50 & 2.0 \\
SLN-C80 (10\%) & $1: 9$ & 2.0 & Carplex-80 & 1.11 & 2.0 \\
SLN-C80 (20\%) & $2: 8$ & 2.0 & Carplex-80 & 2.50 & 2.0 \\
SLN-FPS (10\%) & $1: 9$ & 2.0 & Carplex-FPS 500 & 1.11 & 2.0 \\
SLN-FPS (20\%) & $2: 8$ & 2.0 & Carplex-FPS 500 & 2.50 & 2.0 \\
\hline
\end{tabular}

linder of about $10 \mathrm{~mm}$ in diameter and the unsettled volume was recorded. The cylinder containing powders was then tapped 250 times [30] manually and the volume was recorded again. The Carr's index (CI) and Hausner ratio (HR) was calculated using the following equation:

$$
\begin{aligned}
& \text { Carr's index }=100 \times\left(\rho_{T}-\rho_{B}\right) / \rho_{T} \\
& \text { Hausner ratio }=\rho_{T} / \rho_{B}
\end{aligned}
$$

where, $\rho_{\mathrm{T}}$ and $\rho_{\mathrm{B}}$ represent the tapped density and bulk density, respectively. Both the tests were repeated 3 times.

USP categorize powders according to the scale of flowability depending on the CI and HR value as, $\leq 10$ and 1.00 - 1.11: Excellent; 11 - 15 and 1.12 - 1.18: Good; 16 - 20 and 1.19 - 1.25: Fair; 21 - 25 and 1.26 - 1.34: Passable; 26 - 31 and 1.35 - 1.45: Poor; 32 - 37 and 1.46 - 1.59: Very poor; and $>38$ and >1.60: Very, very poor, respectively.

\subsubsection{Scanning Electron Micrographs (SEM)}

Scanning electron micrographs (SEM) of the SNCs were obtained using a scanning electron microscope (SSX500, Shimadzu, Tokyo, Japan) after platinum metallization. An accelerating voltage of $15 \mathrm{kV}$ was used.

\subsubsection{Mean Particle Size (PS), Polydispersibility Index (PDI), and Zeta Potential (ZP)}

De-ionized distilled water $(2 \mathrm{ml})$ filtered through a membrane filter $(0.20 \mu \mathrm{m})$ was added to the vial containing the freeze-dried samples, and the vial was shaken by vortex agitation to rehydrate them. The PS and PDI were measured by dynamic light scattering (Nano ZS, Malvern Instruments, Worcestershire, UK), and ZP was estimated on the basis of electrophoretic mobility under an electric field at room temperature. The PS was analyzed based on weight distribution.

\subsubsection{Recovered Potency}

The freeze dried samples were re-constituted as described in section 2.3.3. Exactly $0.2 \mathrm{ml}$ of reconstituted sample was taken in an eppendorf tube $(1.5 \mathrm{ml})$ and $0.8 \mathrm{ml}$ of methanol was added followed by sonication for 3 min. It was then centrifuged at $7000 \mathrm{rpm}$ for $10 \mathrm{~min}$. The supernatant was filtered through a $0.2 \mu \mathrm{m}$ syringe filter. The drug content was assayed by RP-HPLC (Shimadzu, Tokyo, Japan) in a binary mode (dual pump), with a UV detector at a wavelength of $236 \mathrm{~nm}$. The HPLC column was reversed phase (Cadenza 5CD-C18, $4 \mathrm{~mm}$ ID $\times$ $150 \mathrm{~mm}$; Imtakt) at $40^{\circ} \mathrm{C}$. The mobile phase of methanol: aqueous formic acid $(0.2 \% \mathrm{v} / \mathrm{v})$ at a ratio of $60: 40$ was delivered at a flow rate of $0.4 \mathrm{ml} / \mathrm{min}$. The retention time of NI was found to be $6.1 \pm 0.1 \mathrm{~min}$. A calibration curve with at least three standard concentrations $(10,50$, and $100 \mu \mathrm{g} / \mathrm{ml})$ of NI was used.

\subsubsection{Aqueous re-Dispersibility Study}

Aqueous re-dispersibility study of the lyophilized samples was performed by mixing both freeze-dried SLN (control) and SNCs equivalent to $190 \mu \mathrm{g}$ of NI in $10 \mathrm{ml}$ of water contained in a $20 \mathrm{ml}$ beaker, stirred conti- 
nuously at $100 \mathrm{rpm}$ at room temperature. The analytic samples ( $0.3 \mathrm{ml}$ each) were withdrawn at intervals of 1,5 , $10,20,30,60,90$, and $120 \mathrm{~min}$, followed by the replacement of an equal volume of water. The solution was then filtered through a membrane filter $(0.45 \mu \mathrm{m})$ to remove any aggregates of silica nanocomposites. The amount of NI dispersed in the solution was monitored using the HPLC method as described in Section 2.3.4 and the ratio of NI released (\%) was calculated.

\subsection{Statistics}

Statistical analyses were performed using the Student's $t$-test with the probability value, $\mathrm{p}<0.05$ indicating significant difference.

\section{Result and Discussion}

In this study, silica nanocomposites were prepared using two hydrophilic fumed silica, Aerosil ${ }^{\circledR} 200$, and Aerosil $^{\circledR} 380$ and three hydrophilic precipitated silica, Carplex ${ }^{\circledR} 67$, Carplex ${ }^{\circledR} 80$, and Carplex ${ }^{\circledR}$ FPS 500. Among them Aerosil ${ }^{\mathbb{B}} 200$ was a nonporous silica. Table 2 represents the powder properties of the silica itself including mean particle size, specific surface area, and tapped density according to the certificate of analysis supplied by the manufacturing origin. In addition, the ZP values of aqueous dispersion $(0.125 \% \mathrm{w} / \mathrm{v})$ of each type of silica are also presented in Table 2. Figure 1 illustrates the differences in molecular properties between fumed and precipitated silica; precipitated silica (Carplex ${ }^{\circledR}$ ) (Figure 1(a)) has a larger number of H-bond acceptors than that of fumed silica (Aerosil ${ }^{\circledR}$ ) (Figure 1(b)). Therefore, the opportunity for the formation of any chemical bonding between silica and SLN is considered to be greater by precipitated silica.

Figure 2(a) and Figure 2(b) show the values of Carr's Index (CI) and Hausner ratio (HR) of the SNCs containing 20\% of SLN, respectively. The CI value of SLN (control) showed 26.5; the smallest value of CI (12.78) was attributed to SLN-C80; and the highest value (37.47) was from SLN-A200. In addition, Figure 2(b) showed the HR value of SLN-C80, as lowest (1.14) and SLN-A200 as highest (1.59). Therefore, the use of silica in the preparation of the nanocomposite improved the overall powder flow characteristics significantly to reach-

Table 2. Powder and electrostatic properties of silica.

\begin{tabular}{ccccc}
\hline Name of Silica & $\begin{array}{c}\text { Mean Particle Size (Coulter } \\
\text { Counter), }(\mu \mathrm{m})^{a}\end{array}$ & $\begin{array}{c}\text { Specific Surface Area } \\
\left(\mathrm{m}^{2} / \mathrm{g}\right)^{a}\end{array}$ & $\begin{array}{c}\text { Tapped Density } \\
(\mathrm{g} / \mathrm{l})^{a}\end{array}$ & $\begin{array}{c}\text { Zeta Potential } \\
(\mathrm{mV})^{b}\end{array}$ \\
\hline Aerosil $^{\circledR} 200$ & 12.0 & 200 & 50 & -26.4 \\
Aerosil $^{\circledR} 380$ & 7.0 & 380 & 50 & -23.7 \\
Carplex $^{\circledR} 67$ & 7.5 & 380 & 140 & -30.4 \\
Carplex $^{\circledR} 80$ & 8.0 & 200 & 145 & -31.2 \\
Carplex $^{\circledR}$ FPS500 & 2.5 & 500 & 155 & -28.5 \\
\hline
\end{tabular}

${ }^{a}$ Values taken from the certificates of analysis supplied by the manufacturer of silica; ${ }^{b}$ Values were obtained using the method described in section 2.3.3 using $0.125 \% \mathrm{w} / \mathrm{v}$ dispersion of silica.

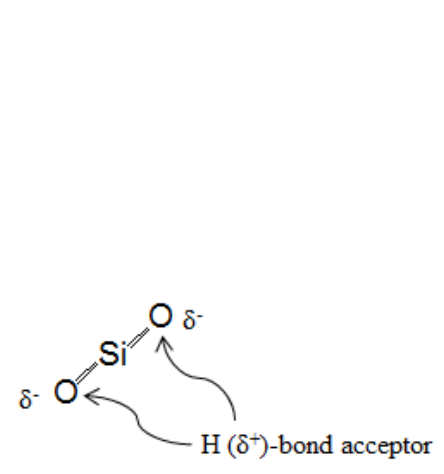

(a)

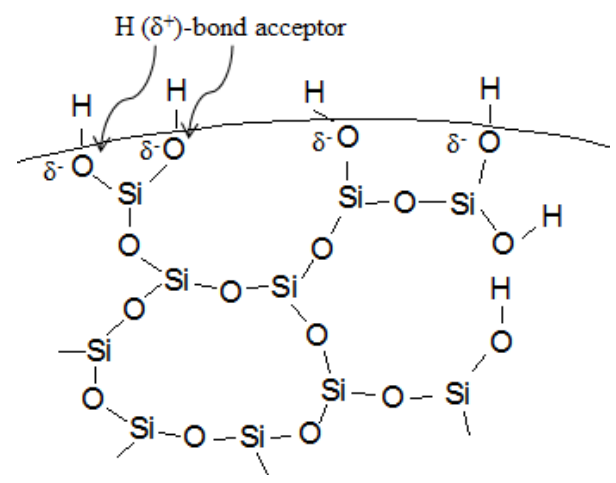

(b)

Figure 1. Chemical structure of silica, (a) Aerosil ${ }^{\circledR}$ and (b) Carplex ${ }^{\circledR}$, highlighting the difference in hydrogen bond acceptors. 


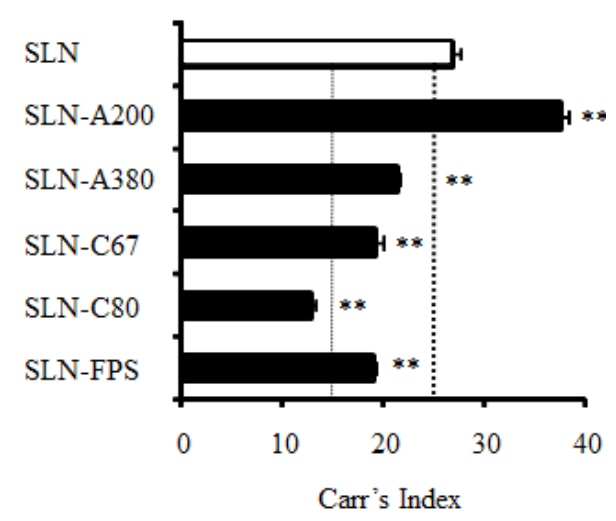

(a)

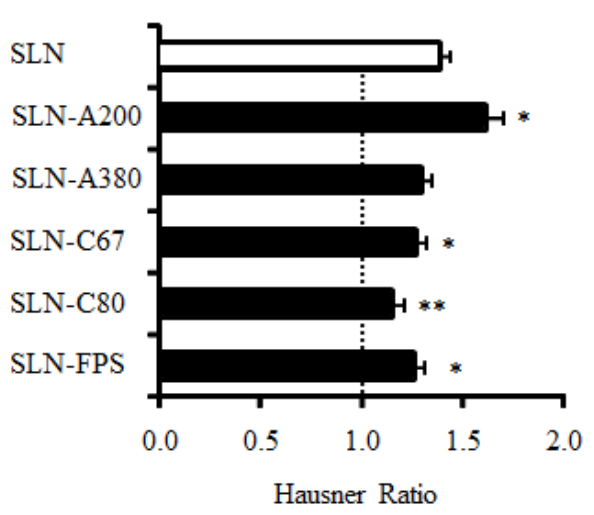

(b)

Figure 2. Powder flow property of freeze dried silica nanocomposites (a) Carr's compressibility index, and (b) Hausner ratio. SLN: Nifedipine solid lipid nanoparticle (NI-SLN) lyophilized with 2.5\% w/w trehalose; SLN-A200: SLN with Aerosil-200 (20\%); SLN-A380: SLN with Aerosil-380 (20\%); SLN-C67: SLN with Carplex-67 (20\%); SLN-C80: SLN with Carplex-80 (20\%); SLN-FPS: SLN with Carplex FPS-500 (20\%). Each value represents the mean $\pm \operatorname{SD}(\mathrm{n}=3) ;{ }^{*} \mathrm{p}<0.05,{ }^{* *} \mathrm{p}<0.01$ versus each value of SLN (control).

from the "passable” to "good” categories when compared with lyophilized SLN, with the exception of Aerosil ${ }^{\circledR} 200$. This may be due to the non-porous nature of this silica leading to the inadequate adsorption of SLNs on its surface. Also, as another reason, tapped density of silica may be involved in this effect; namely, all nanocmposites with Aerosil $^{\circledR}$, having smaller tapped density value, showed the higher CI and HR values than that with Carplex ${ }^{\circledR}$, having larger tapped density value. Among these SNCs, SLN-C80, SLN-F500, and SLN-C67 indicated "good" and "fair" flowability, respectively. The physical properties shown in Table 2 implied that the tapped density of silica as a vehicle might be critically important for the flowability of its nanocomposites. But, the reason of fair flowability of nanocomposites with Carplex ${ }^{\circledR}$ FPS500 although having higher tapped density might be due to poor adsorption characteristics of SLN by silica.

The solid-state surface morphology in the SEM images (Figure 3) showed a three-dimensional internal network of SNCs forming aggregates. Among the Aerosil ${ }^{\circledR}$ nanocomposites, the surface of SLN-A200 was more uneven than SLN-A380. This aligns with the HR and CI values obtained for SLN-A200, which had less flowability than SLN-A380 (Figure 2). On the other hand the regular spherical shaped microparticles with smooth surface were observed by Carplex ${ }^{\circledR}$ nanocomposites, which can be defined as powders with more flowability than the Aerosil ${ }^{\circledR}$ nanocomposites by using CI and HR values. Notably, of the Carplex ${ }^{\circledR}$ nanocomposites, more smooth surfaces of particles seemed to be present in SLN-C80. As the sphericity and surface smoothness of a particle are essential criteria of a powder to be flowable, this SEM results further proved that precipitated silica has the efficiency to increase the flow property of a nanocomposite entity.

The inclusion of silica has the potential to significantly affect the physicochemical parameters of the SLNs, such as PS, PDI, ZP, and recovered concentration due to possible interactions between SLNs and silica. Notably, these parameters are indicators for the long storage stability of nanocomposites. As shown in Figure 4, the PS $(\mathrm{nm})$ of the SNCs was found comparatively smaller with higher silica-to-lipid ratio (2:8) and vice versa, with the exception of the nanocomposite formed by Carplex FPS ${ }^{\circledR} 500$. Especially, among the SNCs, SLN-A200 (20\%), SLN-C80 (20\%), SLN-FPS (10\%), and SLN-C67 (20\%) exhibited PS below $100 \mathrm{~nm}$. In addition, the PDI values in Figure 5 stated more uniform particle distribution (PDI < 0.3) in SLN-C67 (10\%) and SLN-A380 (20\%). However, although these values of nanocomposites were slightly higher than that of SLN, this might be owing to the presence of released SLN from the composites. Given that released SLN and silica co-existed in the solutions, wider particle size distributions might be observed.

$\mathrm{ZP}$ is responsible for the aggregation of particles in storage. Previously, electrostatic stabilization of SLNs was reported to require a $\mathrm{ZP} \geq 30 \mathrm{mV}$ or $\leq-30 \mathrm{mV}$. It has also been reported that a $\mathrm{ZP}$ between 5 and $15 \mathrm{mV}$ results in limited flocculation and a ZP between -5 and $-15 \mathrm{mV}$ leads to maximum flocculation [31]. The SLN itself showed a ZP around $-50 \mathrm{mV}$ (Figure 6). The silica entities, themselves, used to prepare nanocomposites also showed their ZP values around $-25 \mathrm{mV}$ by Aerosil ${ }^{\circledR}$, and $-30 \mathrm{mV}$ by Carplex ${ }^{\circledR}$ (Table 2). Nevertheless, no silica component played an unfavorable role for noticeable change in ZP of SNCs. Almost all SNCs showed 


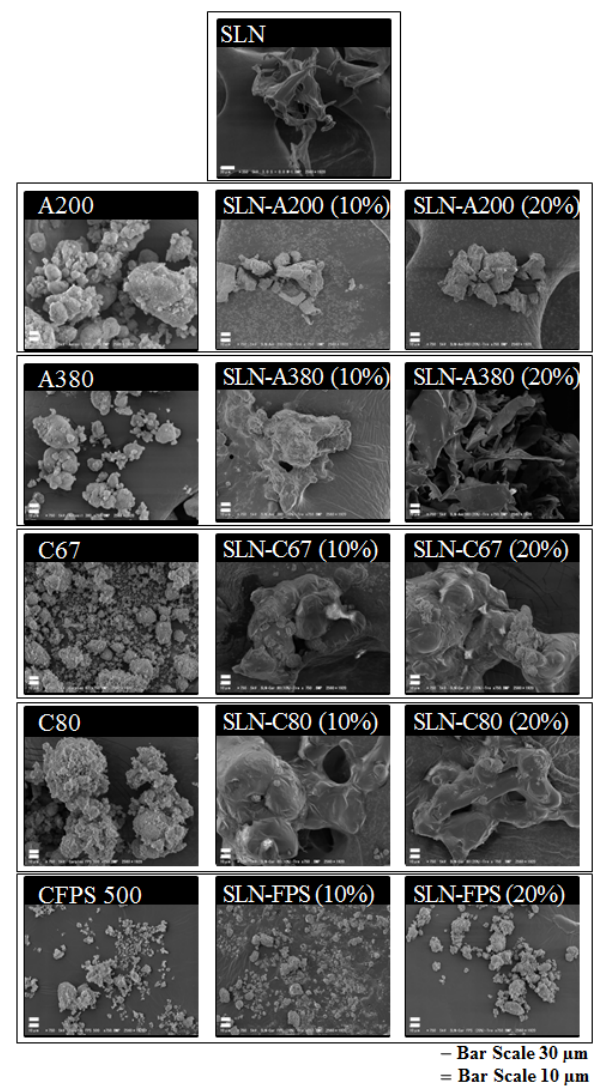

Figure 3. SEM images. SLN: Nifedipine solid lipid nanoparticle (NI-SLN) lyophilized with 2.5\% w/w trehalose; A200: Aerosil-200; A380: Aerosil-380; C67: Carplex-67; C80: Carplex80; CFPS: Carplex-FPS 500; SLN-A200 (10\%) or (20\%): SLN with Aerosil-200 (10\% or $20 \%)$; SLN-A380 (10\%) or (20\%): SLN with Aerosil-380 (10\% or 20\%); SLN-C67 (10\%) or (20\%): SLN with Carplex-67 (10\% or 20\%); SLN-C80 (10\%) or (20\%): SLN with Carplex-80 (10\% or $20 \%)$; SLN-FPS (10\%) or (20\%): SLN with Carplex FPS-500 (10\% or $20 \%$ ), respectively. All silica nanocomposite samples were lyophilized with $2.5 \% \mathrm{w} / \mathrm{v}$ trehalose and freeze dried.

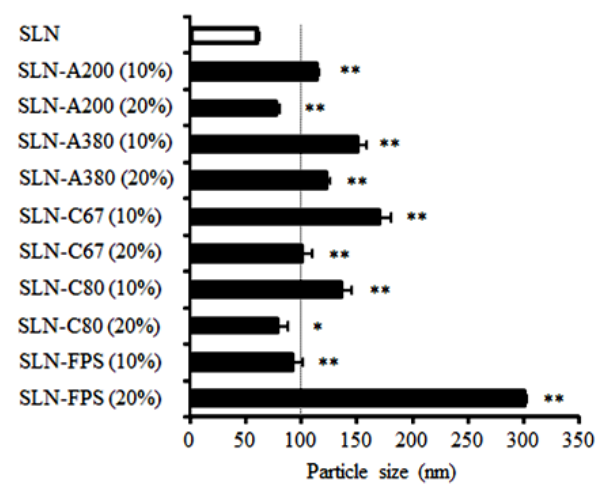

Figure 4. Mean particle size of silica nanocomposites. SLN: Nifedipine solid lipid nanoparticle (NI-SLN) lyophilized with 2.5\% w/w trehalose (as control); SLN-A200 (10\%) or (20\%): SLN with Aerosil-200 (10\% or 20\%); SLN-A380 (10\%) or (20\%): SLN with Aerosil-380 (10\% or $20 \%)$; SLN-C67 (10\%) or (20\%): SLN with Carplex-67 (10\% or $20 \%)$; SLN-C80 (10\%) or (20\%): SLN with Carplex-80 (10\% or $20 \%)$; SLN-FPS $(10 \%)$ or $(20 \%)$ : SLN with Carplex FPS-500 (10\% or $20 \%)$, respectively. The control and all silica nanocomposite samples were lyophilized with $2.5 \% \mathrm{w} / \mathrm{v}$ trehalose and freeze dried. Each value represents the mean $\pm \mathrm{SD},(\mathrm{n}=3) ;{ }^{*} \mathrm{p}<0.05,{ }^{* *} \mathrm{p}<0.01$ versus each value of SLN (control). 


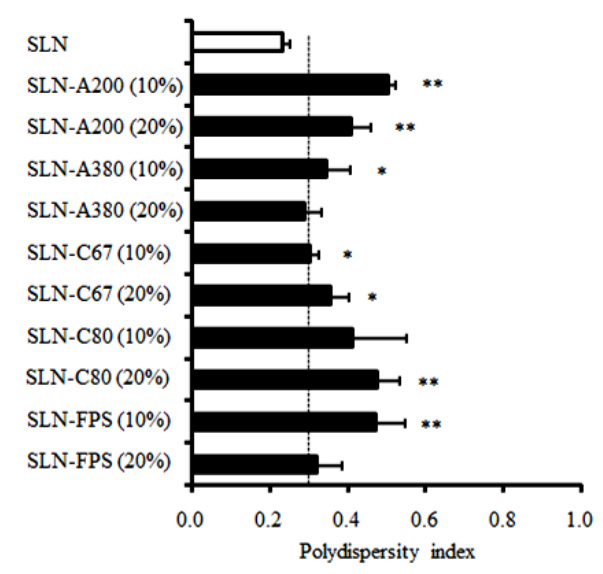

Figure 5. Polydispersity index (PDI) of silica nanocomposites. SLN: Nifedipine solid lipid nanoparticle (NI-SLN) lyophilized with $2.5 \% \mathrm{w} / \mathrm{w}$ trehalose (as control); SLN-A200 (10\%) or (20\%): SLN with Aerosil-200 (10\% or $20 \%)$; SLN-A380 (10\%) or (20\%): SLN with Aerosil$380(10 \%$ or $20 \%)$; SLN-C67 (10\%) or (20\%): SLN with Carplex-67 (10\% or $20 \%)$; SLN-C80 $(10 \%)$ or $(20 \%)$ : SLN with Carplex-80 $(10 \%$ or $20 \%)$; SLN-FPS $(10 \%)$ or $(20 \%)$ : SLN with Carplex FPS-500 (10\% or $20 \%)$, respectively. The control and all silica nanocomposite samples were lyophilized with $2.5 \% \mathrm{w} / \mathrm{v}$ trehalose and freeze dried. Each value represents the mean $\pm \mathrm{SD}(\mathrm{n}=3) ;{ }^{*} \mathrm{p}<0.05,{ }^{* *} \mathrm{p}<0.01$ versus each value of SLN (control).

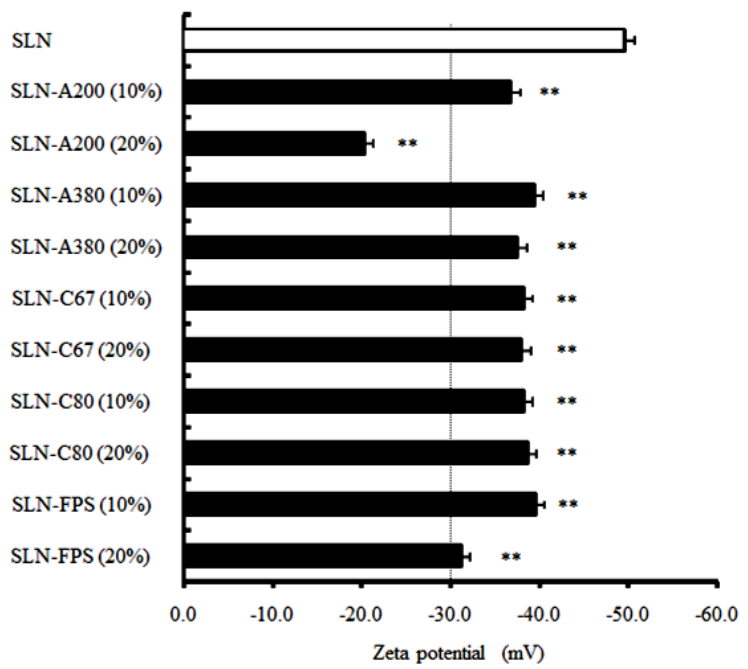

Figure 6. Zeta potential (ZP) of silica nanocomposites. SLN: Nifedipine solid lipid nanoparticle (NI-SLN) lyophilized with 2.5\% w/w trehalose (as control); SLN-A200 (10\%) or (20\%): SLN with Aerosil-200 (10\% or 20\%); SLN-A380 (10\%) or (20\%): SLN with Aerosil-380 (10\% or $20 \%)$; SLN-C67 (10\%) or (20\%): SLN with Carplex-67 (10\% or $20 \%)$; SLN-C80 $(10 \%)$ or $(20 \%)$ : SLN with Carplex-80 $(10 \%$ or $20 \%)$; SLN-FPS $(10 \%)$ or $(20 \%)$ : SLN with Carplex FPS-500 (10\% or $20 \%)$, respectively. The control and all silica nanocomposite samples were lyophilized with $2.5 \% \mathrm{w} / \mathrm{v}$ trehalose and freeze dried. Each value represents the mean $\pm \operatorname{SD}(\mathrm{n}=3) ;{ }^{*} \mathrm{p}<0.05,{ }^{* *} \mathrm{p}<0.01$ versus each value of SLN (control).

their ZP values from -30 to $-50 \mathrm{mV}$ indicating high stability, with the exception of SLN-A200 (20\%).

It is important that the recovered potency of a drug is responsible for the desired level of drug release. All the SNC samples showed high drug recovery, i.e., above 80\% (Figure 7). It might be easy to understand that different silica might have different degree of bonding capacity with solid-lipid nanoparticles resulting in variation of drug recovery. In addition, the re-dispersibility study of SLN (control) and SNCs were also investigated under ambient condition using water as a medium (Figure 8). All samples showed rapid dissolution ratios and the values remained constant, indicating that around 60\% NI-SLNs were easily and rapidly released from SNCs. 


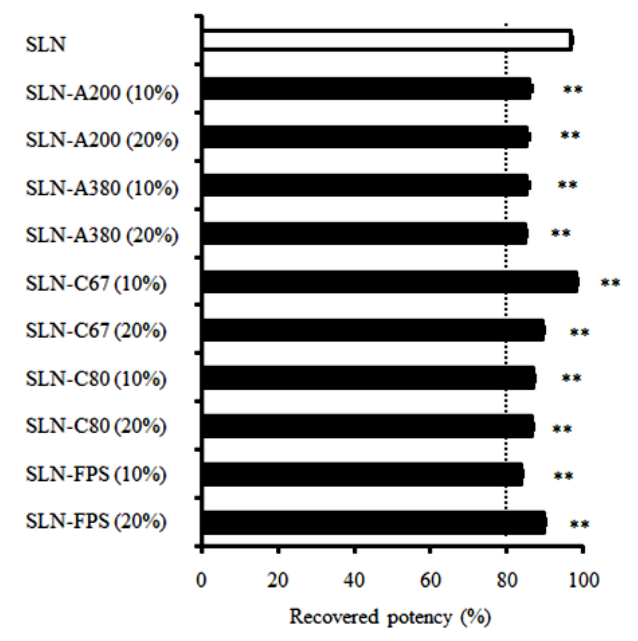

Figure 7. Recovered potency (\%) of silica nanocomposites. SLN: Nifedipine solid lipid nanoparticle (NI-SLN) lyophilized with $2.5 \% \mathrm{w} / \mathrm{w}$ trehalose (as control); SLN-A200 (10\%) or (20\%): SLN with Aerosil-200 (10\% or $20 \%)$; SLN-A380 (10\%) or (20\%): SLN with Aerosil$380(10 \%$ or $20 \%)$; SLN-C67 (10\%) or (20\%): SLN with Carplex-67 (10\% or $20 \%)$; SLN-C80 (10\%) or $(20 \%)$ : SLN with Carplex-80 $(10 \%$ or $20 \%)$; SLN-FPS $(10 \%)$ or $(20 \%)$ : SLN with Carplex FPS-500 (10\% or 20\%), respectively. The control and all silica nanocomposite samples were lyophilized with $2.5 \% \mathrm{w} / \mathrm{v}$ trehalose and freeze dried. Each value represents the mean \pm SD $(\mathrm{n}=3) ;{ }^{*} \mathrm{p}<0.05,{ }^{* *} \mathrm{p}<0.01$ versus each value of SLN (control).

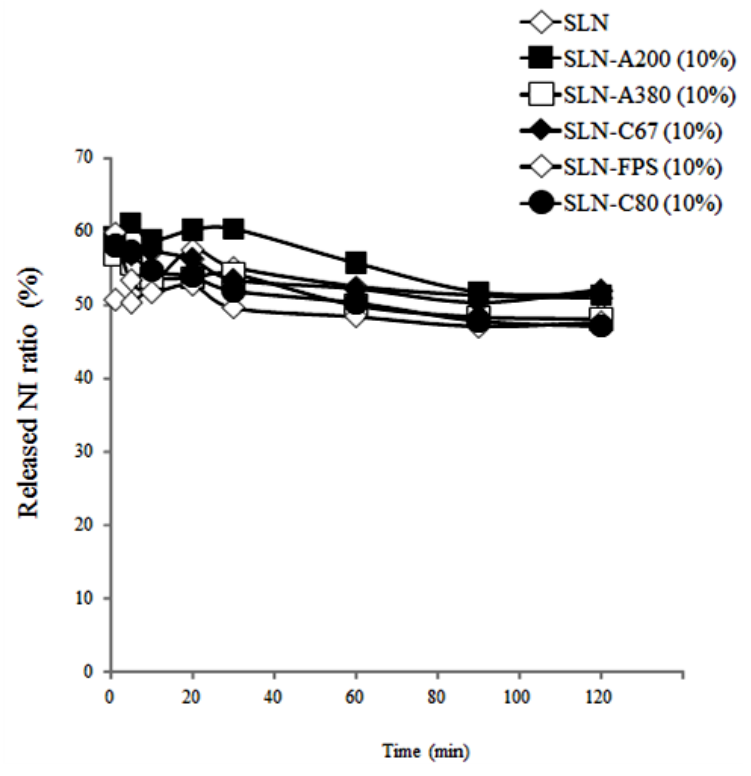

Figure 8. Re-dispersibility profile of silica nanocomposites. SLN: Nifedipine solid lipid nanoparticle (NI-SLN) lyophilized with 2.5\% w/w trehalose (as control); SLN-A200 (10\%) or (20\%): SLN with Aerosil-200 (10\% or 20\%); SLN-A380 (10\%) or (20\%): SLN with Aerosil$380(10 \%$ or $20 \%)$; SLN-C67 (10\%) or (20\%): SLN with Carplex-67 (10\% or 20\%); SLN-C80 $(10 \%)$ or $(20 \%)$ : SLN with Carplex-80 $(10 \%$ or $20 \%)$; SLN-FPS $(10 \%)$ or $(20 \%)$ : SLN with Carplex FPS-500 (10\% or $20 \%)$, respectively. The control and all silica nanocomposite samples were lyophilized with $2.5 \% \mathrm{w} / \mathrm{v}$ trehalose and freeze dried. Each value represents the mean $\pm \operatorname{SD}(n=3)$.

Previously, we reported that NI-SLN showed an increase in the 4-fold $C_{\max }$ compared with NI pure drug; that is to say, enhanced drug absorption may have been observed in SNCs because SLNs were released to be intact form. 


\section{Conclusion}

We successfully improved the flow property of powdered nanoparticles by formulating nanocomposite with various types of silica. The precipitated silica, Carplex ${ }^{\circledR}$, and especially Carplex ${ }^{\circledR} 80$ was found to produce the most favorable results, i.e., with the greatest improvement in flowability and lack of deterioration during drug release. Therefore, this study suggests that Carplex ${ }^{\circledR}$ could be a novel excipient for use in pharmaceutical research and in the development of oral solid dosage form. Further work investigating the long-term stability of this new formulation is essential in the development of an established dosage form. Also a clinical trial would be essentially required to investigate how this new formulation shows the pharmacodynamics.

\section{Acknowledgements}

This investigation was possible owing to a research grant from the Japan Society for the Promotion of Science (JSPS). Therefore the authors would like to cordially acknowledge the JSPS for arranging such a grant through the RONPAKU program. Also, the authors thank Evonik Japan industries Ltd. for kindly providing some types of silica used in this study.

\section{References}

[1] Lipinski, C.A., Lombardo, F., Dominy, B.W. and Feeney, P.J. (2001) Experimental and Computational Approaches to Estimate Solubility and Permeability in Drug Discovery and Development Settings. Advanced Drug Delivery Reviews, 46, 3-26. http://dx.doi.org/10.1016/S0169-409X(00)00129-0

[2] Fahr, A. and Liu, X. (2007) Drug Delivery Strategies for Poorly Water-Soluble Drugs. Expert Opinion on Drug Delivery, 4, 403-416. http://dx.doi.org/10.1517/17425247.4.4.403

[3] Dressman, J. and Christos, R. (2007) Drug Solubility: How to Measure It, How to Improve It. Advanced Drug Delivery Reviews, 59, 531-532. http://dx.doi.org/10.1016/j.addr.2007.05.014

[4] Merisko-Liversidge, E., Liversidge, G.G. and Cooper, E.R. (2003) Nanosizing: A Formulation Approach for Poorly Water-Soluble Compounds. European Journal of Pharmaceutical Sciences, 18, 113-120. http://dx.doi.org/10.1016/S0928-0987(02)00251-8

[5] Müller, R.H., Jacobs, C. and Kayser, O. (2001) Nanosuspensions as Particulate Drug Formulations in Therapy. Rationale for Development and What We Can Expect for the Future. Advanced Drug Delivery Reviews, 47, 3-19. http://dx.doi.org/10.1016/S0169-409X(00)00118-6

[6] Hauss, D.J. (2007) Oral Lipid-Based Formulations. Advanced Drug Delivery Reviews, 59, 667-676. http://dx.doi.org/10.1016/j.addr.2007.05.006

[7] Cole, E.T., Cade, D. and Benameur, H. (2008) Challenges and Opportunities in the Encapsulation of Liquid and SemiSolid Formulations into Capsules for Oral Administration. Advanced Drug Delivery Reviews, 60, 747-756. http://dx.doi.org/10.1016/j.addr.2007.09.009

[8] Leuner, C. and Dressman, J. (2000) Improving Drug Solubility for Oral Delivery Using Solid Dispersions. European Journal of Pharmaceutics and Biopharmaceutics, 50, 47-60. http://dx.doi.org/10.1016/S0939-6411(00)00076-X

[9] Vasconcelos, T., Sarmento, B. and Costa, P. (2007) Solid Dispersions as Strategy to Improve Oral Bioavailability of Poor Water Soluble Drugs. Drug Discovery Today, 12, 1068-1075. http://dx.doi.org/10.1016/j.drudis.2007.09.005

[10] Suri, S.S., Fenniri, H. and Singh, B. (2007) Nanotechnology-Based Drug Delivery Systems. Journal of Occupational Medicine and Toxicology, 2, 16. http://dx.doi.org/10.1186/1745-6673-2-16

[11] Bunjes, H., Westesen, K. and Koch M.H.J. (1996) Cystallization Tendency and Polymorphic Transitions in TriglyceRide Nanoparticles. International Journal of Pharmaceutics, 129, 159-173. http://dx.doi.org/10.1016/0378-5173(95)04286-5

[12] Zimmermann, E., Müller, R.H. and Mader, K. (2000) Influence of Different Parameters on Reconstitution of Lyophilized SLN. International Journal of Pharmaceutics, 196, 211-213. http://dx.doi.org/10.1016/S0378-5173(99)00424-X

[13] Ohshima, H., Miyagishima, A., Kurita, T., Makino, Y., Iwao, Y., Sonobe, T. and Itai, S. (2009) Freeze-Dried Nifedipine-Lipid Nanoparticles with Long-Term Nano-Dispersion Stability after Reconstitution. International Journal of Pharmaceutics, 377, 180-184. http://dx.doi.org/10.1016/j.ijpharm.2009.05.004

[14] Barman, R.K., Iwao, Y., Funakoshi, Y., Ranneh, A.H., Noguchi, S., Wahed, M.I.I. and Itai, S. (2014) Development of Highly Stable Nifedipine Solid-Lipid Nanoparticles. Chemical and Pharmaceutical Bulletin, 62, 399-406. http://dx.doi.org/10.1248/cpb.c13-00684

[15] Barman, R.K., Iwao, Y., Islam, M.R., Funakoshi, Y., Noguchi, S., Wahed, M.I.I. and Itai, S. (2014) In Vivo Pharmaco- 
kinetic and Hemocompatible Evaluation of Lyophilization Induced Nifedipine Solid-Lipid Nanoparticle. Journal of Pharmacy and Pharmacology, 5, 455-461. http://dx.doi.org/10.4236/pp.2014.55055

[16] Funakoshi, Y., Iwao, Y., Noguchi, S. and Itai, S. (2013) Lipid Nanoparticles with No Surfactant Improve Oral Absorption Rate of Poorly Water-Soluble Drug. International Journal of Pharmaceutics, 451, 92-94. http://dx.doi.org/10.1016/j.ijpharm.2013.04.050

[17] Vallet-Regi, M., Ramila, A., Del Real, R. and Perez-Pariente J. (2001) A New Property of MCM-41: Drug Delivery System. Chemistry of Materials, 13, 308-311. http://dx.doi.org/10.1021/cm0011559

[18] Fu, T., Lu, J., Guo, L., Zhang, L., Cai, X. and Zhu, H. (2012) Improving Bioavailability of Silybin by Inclusion into SBA-15 Mesoporous Silica Materials. Journal of Nanoscience and Nanotechnology, 12, 3997-4006. http://dx.doi.org/10.1166/jnn.2012.5826

[19] Li, L., Huang, X., Liu, T., Liu, H., Hao, N., Chen, D., Zhang, Y. and Tang, F. (2012) Overcoming Multidrug Resistance with Mesoporous Silica Nanorods as Nanocarrier of Doxorubicin. Journal of Nanoscience and Nanotechnology, 12, 4458-4466. http://dx.doi.org/10.1166/jnn.2012.6198

[20] Moon, D.S. and Lee, J.K. (2012) Tunable Synthesis of Hierarchical Mesoporous Silica Nanoparticles with Radial Wrinkle Structure. Langmuir, 28, 12341-12347. http://dx.doi.org/10.1021/la302145j

[21] Song, B., Wu, C. and Chang, J. (2012) Controllable Delivery of Hydrophilic and Hydrophobic Drugs from Electrospun Poly(Lactic-Co-Glycolic Acid)/Mesoporous Silica Nanoparticles Composite Mats. Journal of Biomedical Materials Research Part B: Applied Biomaterials, 100, 2178-2186. http://dx.doi.org/10.1002/jbm.b.32785

[22] Shen, S., Chow, P.S., Kim, S., Zhu, K. and Tan, R.B.H. (2008) Synthesis of Carboxyl-Modified Rod-Like SBA-15 by Rapid Co-Condensation. Journal of Colloid and Interface Science, 321, 365-372. http://dx.doi.org/10.1016/j.jcis.2008.02.020

[23] Zhang, Y., Zhi, Z., Jiang, T., Zhang, J., Wang, Z. and Wang, S. (2010) Spherical Mesoporous Silica Nanoparticles for Loading and Release of the Poorly Water-Soluble Drug Telmisartan. Journal of Controlled Release, 145, 257-263. http://dx.doi.org/10.1016/j.jconrel.2010.04.029

[24] Yasmin, R., Tan, A., Bremmell, K.E. and Prestidge, C.A. (2014) Lyophilized Silica-Lipid Hybrid Carriers for Poorly Water-Soluble Drugs: Physicochemical and in Vitro Pharmaceutical Investigations. Journal of Pharmaceutical Sciences, 103, 2950-2959. http://dx.doi.org/10.1002/jps.23914

[25] Simovic, S., Heard, P., Hui, H., Song, Y., Peddie, F., Davey, A.K., Lewis, A., Rades, T. and Prestidge, C.A. (2009) Dry Hybrid Lipid-Silica Microcapsules Engineered from Submicron Lipid Droplets and Nanoparticles as a Novel Delivery System for Poorly Soluble Drugs. Molecular Pharmaceutics, 6, 861-872. http://dx.doi.org/10.1021/mp900063t

[26] Tan, A., Simovic, S., Davey, A.K., Rades, T. and Prestidge, C.A. (2009) Silica-Lipid Hybrid (SLH) Microcapsules: A Novel Oral Delivery System for Poorly Soluble Drugs. Journal of Controlled Release, 134, 62-70. http://dx.doi.org/10.1016/j.jconrel.2008.10.014

[27] Perge, L., Robitzer, M., Guillemot, C., Devoisselle, J.M., Quignard, F. and Legrand P. (2012) New Solid Lipid Microparticles for Controlled Ibuprofen Release: Formulation and Characterization Study. International Journal of Pharmaceutics, 422, 59-67. http://dx.doi.org/10.1016/j.ijpharm.2011.10.027

[28] Kamiya, S., Yamada, M., Kurita, T., Miyagishima, A., Arakawa, M. and Sonobe, T. (2008) Preparation and Stabilization of Nifedipine Lipid Nanoparticles. International Journal of Pharmaceutics, 354, 242-247. http://dx.doi.org/10.1016/j.ijpharm.2007.10.049

[29] Carr, R.L. (1965) Evaluating Flow Properties of Solids. Chemical Engineering Journal, 72, 163-168.

[30] United States of Pharmacopeia (USP)-29 and National Formulary (NF)-24: 1174.

[31] Schwarz, C., Mehnert, W., Lucks, J.S. and Muller, R.H. (1994) Solid Lipid Nanoparticles (SLN) for Controlled Drug Delivery. I. Production, Characterization and Sterilization. Journal of Controlled Release, 30, 83-96. http://dx.doi.org/10.1016/0168-3659(94)90047-7 
Scientific Research Publishing (SCIRP) is one of the largest Open Access journal publishers. It is currently publishing more than 200 open access, online, peer-reviewed journals covering a wide range of academic disciplines. SCIRP serves the worldwide academic communities and contributes to the progress and application of science with its publication.

Other selected journals from SCIRP are listed as below. Submit your manuscript to us via either submit@scirp.org or Online Submission Portal.
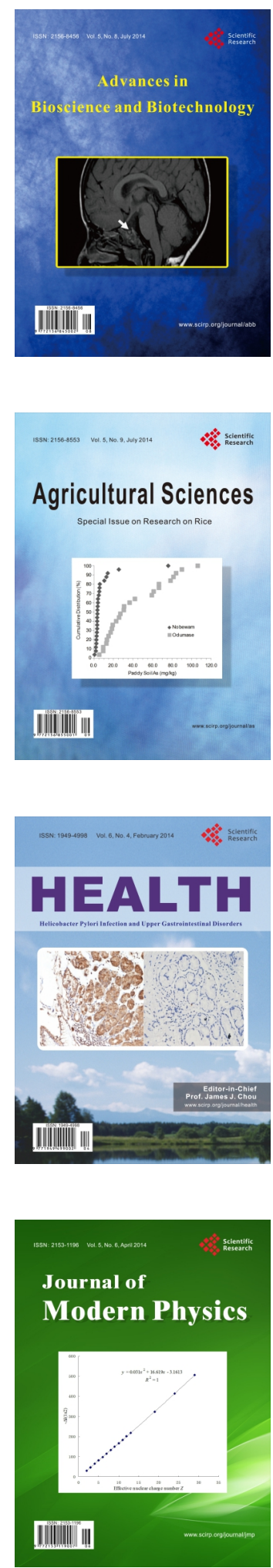
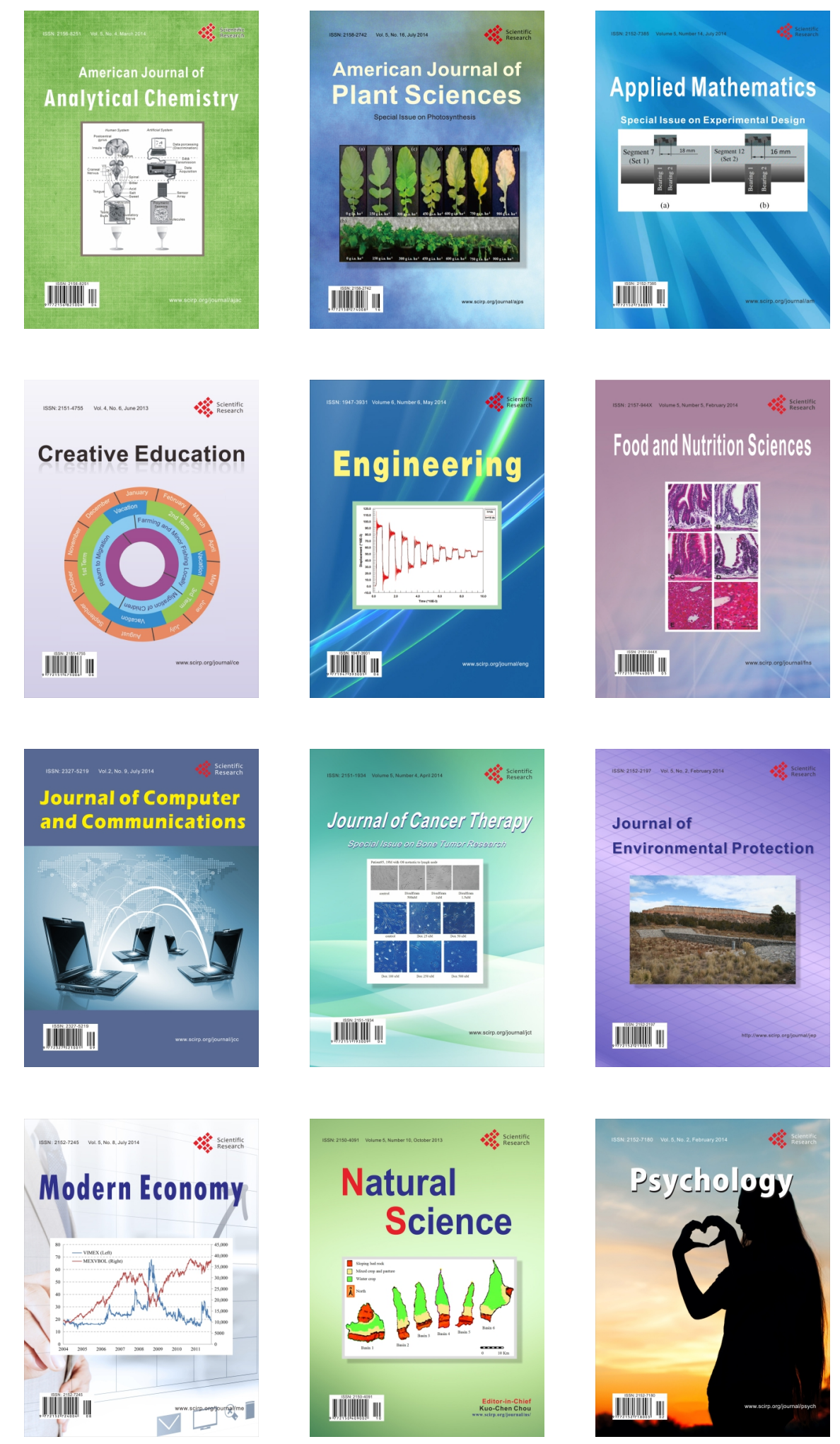